\title{
EDITOR'S CHOICE
}

\section{How science is going sour on sugar}

\author{
Trevor Jackson deputy editor, BMJ
}

When the British physiologist John Yudkin published Pure, White and Deadly — his 1972 book linking heart disease to sugar consumption - he met strong opposition from the sugar industry. As Geoff Watts writes in this week's BMJ (doi:10.1136/bmj. e7800), "jobs and research grants that might predictably have come Yudkin's way did not materialise.” Attacks also included the abrupt cancellation of conferences suspected of promulgating anti-sugar findings, and the book was dismissed as a work of fiction. Enter fat in the role of chief culprit in the rise in heart disease. The fat hypothesis, the chief proponent of which was the American biologist Ancel Keys, influenced policy makers and captured the popular imagination. Meanwhile, writes Watts, medical interest in the sugar hypothesis faded. Yudkin's book fell out of print and low fat became the buzz phrase in nutrition.

But in recent years, and with rising obesity becoming one of the main health concerns in the developed world, the sugar hypothesis has started to regain momentum. Recent anti-sugar initiatives include New York city's restriction on the size of fizzy drinks (BMJ 2012;345:e6768). At the end of last year Penguin Books reissued Pure, White and Deadly, with a new and enthusiastic introduction by US endocrinologist Robert Lustig, which in this week's BMJ Jack Winkler hails as a medical classic (doi:10.1136/bmj.e8612). And, as if to forestall any further policy initiatives against sugary beverages, this week Coca-Cola launched a television advertisement in the United States acknowledging the obesity problem and attempting to defend the company's record in producing low calorie drinks.

Two papers in this week's $B M J$ seem to go right to the heart of the sugar versus fat debate. Lisa Te Morenga and colleagues' systematic review shows an association between the intake of sugars and changes in body weight (doi:10.1136/bmj.e7492), while Lee Hooper and colleagues' systematic review shows that reducing fat intake leads to lower body weight in adults and children (doi:10.1136/bmj.e7666). In an editorial accompanying Te Morenga and colleagues' paper, Walter C Willet and David $\mathrm{S}$ Ludwig acknowledge that the association between sugar and poor health has remained contentious over the past few decades, and attribute this partly to weaknesses in the data and partly to tensions between science and industry-as evidenced by the tale of Pure, White and Deadly (doi:10.1136/bmj.e8077). Welcoming Te Morenga and colleagues' results, which suggest that sugar increases body weight mainly by promoting overconsumption of energy, Willett and Ludwig ask what is a desirable limit for sugar intake and whether it matters what kind of sugar-glucose, fructose, or sucrose - we mean. "No clear threshold exists for the many adverse effects of sugar intake," they say. "In general the association seems to be roughly linear, which makes a limit somewhat arbitrary."

The sugar versus fat debate is far from over, but the pendulum is now definitely swinging away from fat as the root of all evil. Meanwhile, what overall public health message emerges? Willett and Ludwig conclude: "Healthcare providers could play an important role by routinely asking about consumption of sugar sweetened drinks as well as tobacco and alcohol use, by setting a good example, and by assuming leadership in public efforts to limit sugar as a source of harm."

\section{Cite this as: BMJ 2013;346:f307}

๑ BMJ Publishing Group Ltd 2013 